\title{
The one-loop contributions to $c(t)$ electric dipole moment in the CP-violating BLMSSM
}

\author{
Shu-Min Zhao ${ }^{1, \mathrm{a}}$, Tai-Fu Feng ${ }^{1, \mathrm{~b}}$, Zhong-Jun Yang ${ }^{1}$, Hai-Bin Zhang $^{1}$, Xing-Xing Dong ${ }^{1}$, Tao Guo ${ }^{2}$ \\ ${ }^{1}$ Department of Physics, Hebei University, Baoding 071002, China \\ ${ }^{2}$ School of Mathematics and Science, Hebei University of Geosciences, Shijiazhuang 050031, China
}

Received: 25 October 2016 / Accepted: 16 January 2017 / Published online: 14 February 2017

(C) The Author(s) 2017. This article is published with open access at Springerlink.com

\begin{abstract}
In the CP-violating supersymmetric extension of the standard model with local gauged baryon and lepton symmetries (BLMSSM), there are new CP-violating sources which can give new contributions to the quark electric dipole moment (EDM). Considering the CP-violating phases, we analyze the EDMs of the quarks $c$ and $t$. We take into account the contributions from the one-loop diagrams. The numerical results are analyzed with some assumptions on the relevant parameter space. The numerical results for the $c$ and $t$ EDMs can reach large values.
\end{abstract}

\section{Introduction}

The CP-violation found in the K- and B-system [1-3] can be well explained in the standard model. It is well known that the electric dipole moment (EDM) of an elementary particle is a clear signal of CP-violation [4-8]. The Cabbibo-KobayashiMaskawa (CKM) phase is the only source of CP-violation in the SM, which has an ignorable effect on the EDM of the elementary particle. In the SM, even to two-loop order, the EDM of a fermion does not appear, and there are partial cancelations between the three-loop contributions [9-12]. If the EDM of an elementary fermion is detected, one can confirm there are new CP phases and physics beyond the SM.

Though SM has obtained large successes with the detection of the lightest CP-even Higgs $h^{0}[13,14]$, it is unable to explain some phenomena. Physicists consider the SM to be a low energy effective theory of a large model. The minimal supersymmetric extension of the standard model (MSSM) is very favorite and people have been interested in it for a long time [15-17]. There are also many new models beyond the SM, such as $\mu \nu$ SSM $[18,19]$. Generally speaking, the new models introduce new CP-violating phases that

\footnotetext{
a e-mail: zhaosm@hbu.edu.cn

be-mail: fengtf@hbu.edu.cn
}

can affect the EDMs of fermions, $B^{0}-\bar{B}^{0}$ mixing etc. The EDMs of electron and neutron are strict constraints on the CP-violating phases [20-23]. In the models beyond SM, there are new $\mathrm{CP}$-violating phases which can give large contributions to the electron and neutron EDMs [24-26]. To make the MSSM predictions of the electron and neutron EDMs under the experiment upper bounds, there are three possibilities [27,28]: (1) the CP-violating phases are very small, (2) varies contributions cancel with each other in some special parameter spaces, (3) the supersymmetry particles are very heavy at several $\mathrm{TeV}$ order.

Taking into account the local gauged $B$ and $L$, one obtains the minimal supersymmetric extension of the SM, which is the so-called BLMSSM [29,30]. The authors in Refs. [31-33] first proposed BLMSSM, where they studied some phenomena. At TeV scale, the local gauge symmetries of BLMSSM break spontaneously. Therefore, in BLMSSM R-parity is violated and the asymmetry of matter-antimatter in the universe can be explained. We have studied the lightest CP-even Higgs mass and the decays $h^{0} \rightarrow V V, V=(\gamma, Z, W)$ [34] in the BLMSSM, where some other processes [35-37] are also researched. Taking the $\mathrm{CP}$-violating phases with nonzero values, the neutron EDM, the lepton EDM, and $B^{0}-\bar{B}^{0}$ mixing are researched in this model [38-40].

From the experimental neutron data, the bounding of the top EDM is analyzed [41]. Taking into account the precise measurements of the electron and neutron EDMs, the upper limits of heavy quark EDMs are also discussed [42]. The upper limits on the EDMs of heavy quarks are researched from $e^{+} e^{-}$annihilation [43]. In the CP-violating MSSM, the authors studied the $c$ quark EDM including two-loop gluino contributions. There is also other work on the $c$ quark EDM $[44,45]$. Considering the pre-existing work, the upper bounds of the EDMs for $c$ and $t$ are about $d_{c}<5.0 \times 10^{-17} \mathrm{e} \mathrm{cm}$ and $d_{t}<3.06 \times 10^{-15} \mathrm{e} \mathrm{cm}$. In this work, we calculate the EDMs of the charm quark and top quark in the framework of the CP-violating BLMSSM. At the low energy scale, the quark 
chromoelectric dipole moment (CEDM) may give important contributions to the quark EDM. So, we also study the quark CEDM with the renormalization group equations.

In Sect. 2, we briefly introduce the BLMSSM and show the needed mass matrices and couplings, after this introduction. The EDMs (CEDMs) of $c$ and $t$ are researched in Sect. 3. In Sect. 4 , we give the input parameters and calculate the numerical results. The last section is used to discuss the results and the allowed parameter space.

\section{The BLMSSM}

Considering the local gauge symmetries of $B(L)$ and enlarging the local gauge group of the SM to $S U(3)_{C} \otimes S U(2)_{L} \otimes$ $U(1)_{Y} \otimes U(1)_{B} \otimes U(1)_{L}$ one can obtain the BLMSSM model $[29,30]$. In the BLMSSM, there are the exotic superfields including the new quarks $\hat{Q}_{4} \sim\left(3,2,1 / 6, B_{4}, 0\right)$, $\hat{U}_{4}^{c} \sim\left(\overline{3}, 1,-2 / 3,-B_{4}, 0\right), \hat{D}_{4}^{c} \sim\left(\overline{3}, 1,1 / 3,-B_{4}, 0\right)$, $\hat{Q}_{5}^{c} \sim\left(\overline{3}, 2,-1 / 6,-\left(1+B_{4}\right), 0\right), \hat{U}_{5} \sim(3,1,2 / 3,1+$ $\left.B_{4}, 0\right), \hat{D}_{5} \sim\left(3,1,-1 / 3,1+B_{4}, 0\right)$, and the new leptons $\hat{L}_{4} \sim\left(1,2,-1 / 2,0, L_{4}\right), \hat{E}_{4}^{c} \sim\left(1,1,1,0,-L_{4}\right)$, $\hat{N}_{4}^{c} \sim\left(1,1,0,0,-L_{4}\right), \hat{L}_{5}^{c} \sim\left(1,2,1 / 2,0,-\left(3+L_{4}\right)\right)$, $\hat{E}_{5} \sim\left(1,1,-1,0,3+L_{4}\right), \hat{N}_{5} \sim\left(1,1,0,0,3+L_{4}\right)$ to cancel the $B$ and $L$ anomalies.

With the detection of the lightest CP-even Higgs $h^{0}$ at LHC $[13,14]$, the Higgs mechanism is very convincing for particle physics, and BLMSSM is based on the Higgs mechanism. The introduced Higgs superfields $\hat{\Phi}_{L}(1,1,0,0,-2)$, $\hat{\varphi}_{L}(1,1,0,0,2)$ and $\hat{\Phi}_{B}(1,1,0,1,0), \hat{\varphi}_{B}(1,1,0,-1,0)$ break the lepton number and baryon number spontaneously. These Higgs superfields acquire nonzero vacuum expectation values (VEVs) and provide masses to the exotic leptons and exotic quarks. To make the heavy exotic quarks unstable the superfields $\hat{X}\left(1,1,0,2 / 3+B_{4}, 0\right), \hat{X}^{\prime}(1,1,0,-(2 / 3+$ $\left.B_{4}\right), 0$ ) are introduced in the BLMSSM.

The $S U(2)_{L}$ doublets $H_{u}, H_{d}$ obtain nonzero VEVs $v_{u}, v_{d}$,

$$
\begin{gathered}
H_{u}=\left(\begin{array}{c}
H_{u}^{+} \\
\frac{1}{\sqrt{2}}\left(v_{u}+H_{u}^{0}+i P_{u}^{0}\right)
\end{array}\right), \\
H_{d}=\left(\begin{array}{c}
\frac{1}{\sqrt{2}}\left(v_{d}+H_{d}^{0}+i P_{d}^{0}\right) \\
H_{d}^{-}
\end{array}\right) .
\end{gathered}
$$

The $S U(2)_{L}$ singlets $\Phi_{B}, \varphi_{B}$ and $\Phi_{L}, \varphi_{L}$ obtain nonzero $\operatorname{VEVs} v_{B}, \bar{v}_{B}$ and $v_{L}, \bar{v}_{L}$, respectively,

$$
\begin{aligned}
& \Phi_{B}=\frac{1}{\sqrt{2}}\left(v_{B}+\Phi_{B}^{0}+i P_{B}^{0}\right), \varphi_{B}=\frac{1}{\sqrt{2}}\left(\bar{v}_{B}+\varphi_{B}^{0}+i \bar{P}_{B}^{0}\right) . \\
& \Phi_{L}=\frac{1}{\sqrt{2}}\left(v_{L}+\Phi_{L}^{0}+i P_{L}^{0}\right), \varphi_{L}=\frac{1}{\sqrt{2}}\left(\bar{v}_{L}+\varphi_{L}^{0}+i \bar{P}_{L}^{0}\right) .
\end{aligned}
$$

Therefore, the local gauge symmetry $S U(2)_{L} \otimes U(1)_{Y} \otimes$ $U(1)_{B} \otimes U(1)_{L}$ breaks down to the electromagnetic symmetry $U(1)_{e}$.

We write the superpotential of BLMSSM [34]

$$
\begin{aligned}
& \mathcal{W}_{\mathrm{BLMSSM}}=\mathcal{W}_{\mathrm{MSSM}}+\mathcal{W}_{B}+\mathcal{W}_{L}+\mathcal{W}_{X}, \\
& \mathcal{W}_{B}=\lambda_{Q} \hat{Q}_{4} \hat{Q}_{5}^{c} \hat{\Phi}_{B}+\lambda_{U} \hat{U}_{4}^{c} \hat{U}_{5} \hat{\varphi}_{B}+\lambda_{D} \hat{D}_{4}^{c} \hat{D}_{5} \hat{\varphi}_{B} \\
& \quad+\mu_{B} \hat{\Phi}_{B} \hat{\varphi}_{B}+Y_{u_{4}} \hat{Q}_{4} \hat{H}_{u} \hat{U}_{4}^{c}+Y_{d_{4}} \hat{Q}_{4} \hat{H}_{d} \hat{D}_{4}^{c} \\
& \quad+Y_{u_{5}} \hat{Q}_{5}^{c} \hat{H}_{d} \hat{U}_{5}+Y_{d_{5}} \hat{Q}_{5}^{c} \hat{H}_{u} \hat{D}_{5}, \\
& \mathcal{W}_{L}=Y_{e_{4}} \hat{L}_{4} \hat{H}_{d} \hat{E}_{4}^{c}+Y_{v_{4}} \hat{L}_{4} \hat{H}_{u} \hat{N}_{4}^{c}+Y_{e_{5}} \hat{L}_{5}^{c} \hat{H}_{u} \hat{E}_{5} \\
& \quad+Y_{\nu_{5}} \hat{L}_{5}^{c} \hat{H}_{d} \hat{N}_{5}+Y_{v} \hat{L} \hat{H}_{u} \hat{N}^{c}+\lambda_{N^{c}} \hat{N}^{c} \hat{N}^{c} \hat{\varphi}_{L}+\mu_{L} \hat{\Phi}_{L} \hat{\varphi}_{L}, \\
& \mathcal{W}_{X}=\lambda_{1} \hat{Q} \hat{Q}_{5}^{c} \hat{X}+\lambda_{2} \hat{U}^{c} \hat{U}_{5} \hat{X}^{\prime}+\lambda_{3} \hat{D}^{c} \hat{D}_{5} \hat{X}^{\prime}+\mu_{X} \hat{X} \hat{X}^{\prime},
\end{aligned}
$$

with $\mathcal{W}_{\text {MSSM }}$ representing the superpotential of the MSSM. The soft breaking terms $\mathcal{L}_{\text {soft }}$ of the BLMSSM are collected here $[29,30,34]$ :

$$
\begin{aligned}
& \mathcal{L}_{\text {soft }}=\mathcal{L}_{\text {soft }}^{\mathrm{MSSM}}-\left(m_{\tilde{N}^{c}}^{2}\right)_{I J} \tilde{N}_{I}^{c *} \tilde{N}_{J}^{c}-m_{\tilde{Q}_{4}}^{2} \tilde{Q}_{4}^{\dagger} \tilde{Q}_{4} \\
& -m_{\tilde{U}_{4}}^{2} \tilde{U}_{4}^{c *} \tilde{U}_{4}^{c}-m_{\tilde{D}_{4}}^{2} \tilde{D}_{4}^{c *} \tilde{D}_{4}^{c} \\
& -m_{\tilde{Q}_{5}}^{2} \tilde{Q}_{5}^{c \dagger} \tilde{Q}_{5}^{c}-m_{\tilde{U}_{5}}^{2} \tilde{U}_{5}^{*} \tilde{U}_{5}-m_{\tilde{D}_{5}}^{2} \tilde{D}_{5}^{*} \tilde{D}_{5} \\
& -m_{\tilde{L}_{4}}^{2} \tilde{L}_{4}^{\dagger} \tilde{L}_{4}-m_{\tilde{v}_{4}}^{2} \tilde{N}_{4}^{c *} \tilde{N}_{4}^{c} \\
& -m_{\tilde{e}_{4}}^{2} \tilde{E}_{4}^{c *} \tilde{E}_{4}^{c}-m_{\tilde{L}_{5}}^{2} \tilde{L}_{5}^{c \dagger} \tilde{L}_{5}^{c} \\
& -m_{\tilde{v}_{5}}^{2} \tilde{N}_{5}^{*} \tilde{N}_{5}-m_{\tilde{e}_{5}}^{2} \tilde{E}_{5}^{*} \tilde{E}_{5}-m_{\Phi_{B}}^{2} \Phi_{B}^{*} \Phi_{B} \\
& -m_{\varphi_{B}}^{2} \varphi_{B}^{*} \varphi_{B}-m_{\Phi_{L}}^{2} \Phi_{L}^{*} \Phi_{L}-m_{\varphi_{L}}^{2} \varphi_{L}^{*} \varphi_{L} \\
& -\left(m_{B} \lambda_{B} \lambda_{B}+m_{L} \lambda_{L} \lambda_{L}+\text { h.c. }\right) \\
& +\left\{A_{u_{4}} Y_{u_{4}} \tilde{Q}_{4} H_{u} \tilde{U}_{4}^{c}+A_{d_{4}} Y_{d_{4}} \tilde{Q}_{4} H_{d} \tilde{D}_{4}^{c}\right. \\
& +A_{u_{5}} Y_{u_{5}} \tilde{Q}_{5}^{c} H_{d} \tilde{U}_{5}+A_{d_{5}} Y_{d_{5}} \tilde{Q}_{5}^{c} H_{u} \tilde{D}_{5} \\
& +A_{B Q} \lambda_{Q} \tilde{Q}_{4} \tilde{Q}_{5}^{c} \Phi_{B}+A_{B U} \lambda_{U} \tilde{U}_{4}^{c} \tilde{U}_{5} \varphi_{B} \\
& \left.+A_{B D} \lambda_{D} \tilde{D}_{4}^{c} \tilde{D}_{5} \varphi_{B}+B_{B} \mu_{B} \Phi_{B} \varphi_{B}+\text { h.c. }\right\} \\
& +\left\{A_{e_{4}} Y_{e_{4}} \tilde{L}_{4} H_{d} \tilde{E}_{4}^{c}+A_{v_{4}} Y_{v_{4}} \tilde{L}_{4} H_{u} \tilde{N}_{4}^{c}\right. \\
& +A_{e_{5}} Y_{e_{5}} \tilde{L}_{5}^{c} H_{u} \tilde{E}_{5}+A_{\nu_{5}} Y_{\nu_{5}} \tilde{L}_{5}^{c} H_{d} \tilde{N}_{5} \\
& +A_{N} Y_{v} \tilde{L} H_{u} \tilde{N}^{c}+A_{N^{c}} \lambda_{N^{c}} \tilde{N}^{c} \tilde{N}^{c} \varphi_{L} \\
& \left.+B_{L} \mu_{L} \Phi_{L} \varphi_{L}+\text { h.c. }\right\} \\
& +\left\{A_{1} \lambda_{1} \tilde{Q} \tilde{Q}_{5}^{c} X+A_{2} \lambda_{2} \tilde{U}^{c} \tilde{U}_{5} X^{\prime}\right. \\
& \left.+A_{3} \lambda_{3} \tilde{D}^{c} \tilde{D}_{5} X^{\prime}+B_{X} \mu_{X} X X^{\prime}+\text { h.c. }\right\} \text {. }
\end{aligned}
$$




\subsection{Mass matrix}

From the soft breaking terms and the scalar potential, we deduce the mass squared matrix for superfields $X$; we have

$$
\begin{aligned}
-\mathcal{L}_{X} & =\left(\begin{array}{ll}
X^{*} & X^{\prime}
\end{array}\right)\left(\begin{array}{cc}
\left|\mu_{X}\right|^{2}+S_{X} & -\mu_{X}^{*} B_{X}^{*} \\
-\mu_{X} B_{X} & \left|\mu_{X}\right|^{2}-S_{X}
\end{array}\right)\left(\begin{array}{c}
X \\
X^{\prime *}
\end{array}\right), \\
S_{X} & =\frac{g_{B}^{2}}{2}\left(\frac{2}{3}+B_{4}\right)\left(v_{B}^{2}-\bar{v}_{B}^{2}\right) .
\end{aligned}
$$

We diagonalize the mass squared matrix for the superfields $X$ through the unitary transformation,

$$
\begin{aligned}
& \left(\begin{array}{l}
X_{1} \\
X_{2}
\end{array}\right)=Z_{X}^{\dagger}\left(\begin{array}{c}
X \\
X^{\prime *}
\end{array}\right), \\
& Z_{X}^{\dagger}\left(\begin{array}{cc}
\left|\mu_{X}\right|^{2}+S_{X} & -\mu_{X}^{*} B_{X}^{*} \\
-\mu_{X} B_{X} & \left|\mu_{X}\right|^{2}-S_{X}
\end{array}\right) Z_{X}=\left(\begin{array}{cc}
m_{X_{1}}^{2} & 0 \\
0 & m_{X_{2}}^{2}
\end{array}\right) .
\end{aligned}
$$

$\psi_{X}$ and $\psi_{X^{\prime}}$ are the superpartners of the scalar superfields $X$ and $X^{\prime} . \psi_{X}$ and $\psi_{X^{\prime}}$ can composite four-component Dirac spinors, whose mass term are given out [38-40]

$$
-\mathcal{L}_{\tilde{X}}^{\text {mass }}=\mu_{X} \overline{\tilde{X}} \tilde{X}, \quad \tilde{X}=\left(\begin{array}{c}
\psi_{X} \\
\bar{\psi}_{X^{\prime}}
\end{array}\right),
$$

with $\mu_{X}$ denoting the mass of $\tilde{X}$.

In the BLMSSM, there are the new baryon boson, the $S U(2)_{L}$ singlets $\Phi_{B}$ and $\varphi_{B}$. Their superpartners are, respectively, $\lambda_{B}, \psi_{\Phi_{B}}$ and $\psi_{\varphi_{B}}$, and they mix together producing three baryon neutralinos. In the base $\left(i \lambda_{B}, \psi_{\Phi_{B}}, \psi_{\varphi_{B}}\right)$, the mass mixing matrix $M_{B N}$ is obtained and diagonalized by the rotation matrix $Z_{N_{B}}$ [46],

$M_{B N}=\left(\begin{array}{lll}2 m_{B} & -v_{B} g_{B} & \bar{v}_{B} g_{B} \\ -v_{B} g_{B} & 0 & -\mu_{B} \\ \bar{v}_{B} g_{B} & -\mu_{B} & 0\end{array}\right), \quad \chi_{B_{i}}^{0}=\left(\begin{array}{l}k_{B_{i}}^{0} \\ \bar{k}_{B_{i}}^{0}\end{array}\right)$,

$i \lambda_{B}=Z_{N_{B}}^{1 i} k_{B_{i}}^{0}, \quad \psi_{\Phi_{B}}=Z_{N_{B}}^{2 i} k_{B_{i}}^{0}, \quad \psi_{\varphi_{B}}=Z_{N_{B}}^{3 i} k_{B_{i}}^{0}$.

$\chi_{B_{i}}^{0}(i=1,2,3)$ represent the mass eigenstates of the baryon neutralinos.

The exotic quarks with charge $2 / 3$ is written in terms of four-component Dirac spinors, whose mass matrix reads [34]

$$
-\mathcal{L}_{t^{\prime}}^{\text {mass }}=\left(\bar{t}_{4 R}^{\prime} \bar{t}_{5 R}^{\prime}\right)\left(\begin{array}{cc}
\frac{1}{\sqrt{2}} \lambda Q v_{B} & -\frac{1}{\sqrt{2}} Y_{u_{5}} v_{d} \\
-\frac{1}{\sqrt{2}} Y_{u_{4}} v_{u} & \frac{1}{\sqrt{2}} \lambda_{u} \bar{v}_{B}
\end{array}\right)\left(\begin{array}{c}
t_{4 L}^{\prime} \\
t_{5 L}^{\prime}
\end{array}\right) .
$$

Using the unitary transformations, the two mass eigenstates of exotic quarks with charge $2 / 3$ are obtained by the rotation matrices $U_{t}$ and $W_{t}$,

$$
\begin{aligned}
& \left(\begin{array}{c}
t_{4 L} \\
t_{5 L}
\end{array}\right)=U_{t}^{\dagger}\left(\begin{array}{c}
t_{4 L}^{\prime} \\
t_{5 L}^{\prime}
\end{array}\right), \quad\left(\begin{array}{c}
t_{4 R} \\
t_{5 R}
\end{array}\right)=W_{t}^{\dagger}\left(\begin{array}{c}
t_{4 R}^{\prime} \\
t_{5 R}^{\prime}
\end{array}\right), \\
& W_{t}^{\dagger}\left(\begin{array}{cc}
\frac{1}{\sqrt{2}} \lambda_{Q} v_{B} & -\frac{1}{\sqrt{2}} Y_{u_{5}} v_{d} \\
-\frac{1}{\sqrt{2}} Y_{u_{4}} v_{u} & \frac{1}{\sqrt{2}} \lambda_{u} \bar{v}_{B}
\end{array}\right) U_{t}=\operatorname{diag}\left(m_{t_{4}}, m_{t_{5}}\right) .
\end{aligned}
$$

The mass squared matrix for charge $2 / 3$ exotic squarks $\mathcal{M}_{\tilde{t}^{\prime}}^{2}$ is obtained in our previous work [34]. For saving space, we do not show it here. $\mathcal{M}_{\tilde{t}^{\prime}}^{2}$ is diagonalized by $Z_{\tilde{t}^{\prime}}$ through the formula $Z_{\tilde{t}^{\prime}}^{\dagger} \mathcal{M}_{\tilde{t}^{\prime}}^{2} Z_{\tilde{t}^{\prime}}=\operatorname{diag}\left(m_{\tilde{\mathcal{U}}_{1}}^{2}, m_{\tilde{\mathcal{U}}_{2}}^{2}, m_{\tilde{\mathcal{U}}_{3}}^{2}, m_{\tilde{\mathcal{U}}_{4}}^{2}\right)$.

\subsection{Needed couplings}

To study the quark EDMs, the couplings between photon (gluon) and exotic quarks (exotic squarks) are necessary. We derive the couplings between photon (gluon) and exotic quarks, thus

$$
\mathcal{L}_{\gamma(g) q^{\prime} q^{\prime}}=-\frac{2 e}{3} \sum_{i=1}^{2} \bar{t}_{i+3} \gamma^{\mu} t_{i+3} F_{\mu}-g_{3} \sum_{i=1}^{2} \bar{t}_{i+3} T^{a} \gamma^{\mu} t_{i+3} G_{\mu}^{a},
$$

with $F_{\mu}$ and $G_{\mu}^{a}$ representing the electromagnetic field and gluon field, respectively. $T^{a}(a=1, \ldots, 8)$ are the strong $S U$ (3) gauge group generators. Similarly, the couplings between the photon (gluon) and exotic squarks are also deduced

$$
\begin{aligned}
\mathcal{L}_{\gamma(g) \tilde{q}^{\prime} \tilde{q}^{\prime}}= & -\frac{2}{3} e \sum_{j, \beta=1}^{4} \delta_{j \beta} F_{\mu} \tilde{\mathcal{U}}_{j}^{*} i \tilde{\partial}^{\mu} \tilde{\mathcal{U}}_{\beta} \\
& -g_{3} T^{a} \sum_{j, \beta=1}^{4} \delta_{j \beta} G_{\mu}^{a} \tilde{\mathcal{U}}_{j}^{*} i \tilde{\partial}^{\mu} \tilde{\mathcal{U}}_{\beta} .
\end{aligned}
$$

From the superpotential $W_{X}$, one can find there are interactions at tree level for quark, exotic quark and $X$. The needed Yukawa interactions can be deduced from the superpotential $W_{X}$. The quark-exotic quark- $X$ couplings are shown in the mass basis,

$$
\begin{aligned}
& \mathcal{L}_{X t^{\prime} u}=\sum_{i, j=1}^{2}\left(\left(\mathcal{N}_{t^{\prime}}^{L}\right)_{i j} X_{j} \bar{t}_{i+3} P_{L} u^{I}+\left(\mathcal{N}_{t^{\prime}}^{R}\right)_{i j}\right. \\
& \left.\quad \times X_{j} \bar{t}_{i+3} P_{R} u^{I}\right)+ \text { h.c. } \\
& \left(\mathcal{N}_{t^{\prime}}^{L}\right)_{i j}=-\lambda_{1}\left(W_{t}^{\dagger}\right)_{i 1}\left(Z_{X}\right)_{1 j}, \\
& \left(\mathcal{N}_{t^{\prime}}^{R}\right)_{i j}=-\lambda_{2}^{*}\left(U_{t}^{\dagger}\right)_{i 2}\left(Z_{X}\right)_{2 j} .
\end{aligned}
$$

From the superpotential $W_{X}$, in the same way we can also obtain the Yukawa couplings of another type (quark-exotic squark- $\tilde{X}$ ) [38-40]. We have

$$
\mathcal{L}_{\bar{u} \tilde{X} \tilde{\mathcal{U}}}=-\sum_{i=1}^{4} \bar{u}\left(\lambda_{1}\left(Z_{\tilde{t}^{\prime}}\right)_{3 i}^{*} P_{L}+\lambda_{2}\left(Z_{\tilde{t}^{\prime}}\right)_{4 i} P_{R}\right) \tilde{X} \tilde{\mathcal{U}}_{i} .
$$

Beyond the MSSM, there are couplings for the baryon neutralino, quarks, and squarks. They are deduced in our previous work [46], and can give new contributions to the quark EDMs. We have 


$$
\begin{aligned}
& \mathcal{L}\left(\chi_{B}^{0} q \tilde{q}\right)=\sum_{I, i=1}^{3} \sum_{j=1}^{6} \frac{\sqrt{2}}{3} g_{B} \bar{\chi}_{B_{i}^{0}} \\
& \quad \times\left(Z_{N_{B}}^{1 i} Z_{\tilde{U}}^{I j *} P_{L}-Z_{N_{B}}^{1 i *} Z_{\tilde{U}}^{(I+3) j *} P_{R}\right) u^{I} \tilde{U}_{j}^{*}+\text { h.c. }
\end{aligned}
$$

\section{Formulation}

Using the effective Lagrangian [47] method, one obtains the fermion $\mathrm{EDM} d_{f}$ from

$$
\mathcal{L}_{\mathrm{EDM}}=-\frac{i}{2} d_{f} \bar{f} \sigma^{\mu \nu} \gamma_{5} f F_{\mu \nu},
$$

with $F_{\mu \nu}$ representing the electromagnetic field strength and $f$ denoting a fermion field. It is obvious that this effective Lagrangian is CP-violating. In the fundamental interactions, this CP-violating Lagrangian cannot be obtained at tree level. Considering the CP-violating electroweak theory, one can get this effective Lagrangian from the loop diagrams. The chromoelectric dipole moment (CEDM) $\bar{f} T^{a} \sigma^{\mu \nu} \gamma_{5} f G_{\mu \nu}^{a}$ of the quark can also give contribution to the quark EDM. $G_{\mu \nu}^{a}$ denotes the gluon field strength.

To describe the CP-violating operators obtained from the loop diagrams, the effective method is convenient. The coefficients of the quark EDM and CEDM at the matching scale $\mu$ should be evolved down to the quark mass scale with the renormalization group equations. At the matching scale, we can obtain the effective Lagrangian with the CP-violating operators. The effective Lagrangian containing operators relating with the quark EDM and CEDM are

$$
\begin{aligned}
& \mathcal{L}_{\text {eff }}=\sum_{i}^{4} C_{i}(\Lambda) \mathcal{O}_{i}(\Lambda), \\
& \mathcal{O}_{1}=\bar{q} \sigma^{\mu \nu} P_{L} q F_{\mu \nu}, \quad \mathcal{O}_{2}=\bar{q} \sigma^{\mu \nu} P_{R} q F_{\mu \nu}, \\
& \mathcal{O}_{3}=\bar{q} T^{a} \sigma^{\mu \nu} P_{L} q G_{\mu \nu}^{a}, \quad \mathcal{O}_{4}=\bar{q} T^{a} \sigma^{\mu \nu} P_{R} q G_{\mu \nu}^{a},
\end{aligned}
$$

with $\Lambda$ representing the energy scale where the Wilson coefficients $C_{i}(\Lambda)$ are evaluated.

In our previous work [38-40], we have studied the neutron EDM in the CP-violating BLMSSM, where the contributions from baryon neutralino-squark and $\tilde{X}$-exotic squark are neglected, because they are all small in the used parameter space. Here we take into account all the contributions at one-loop level to study the $c$ and $t$ EDMs. In the CPviolating BLMSSM, the one-loop corrections to the quark EDMs and CEDMs can be divided into six types according to the quark self-energy diagrams. We divide the quark selfenergy diagrams according to the virtual particles, thus: (1) gluino-squark, (2) neutralino-squark, (3) chargino-squark, (4) X-exotic quark, (5) baryon neutralino-squark, (6) $\tilde{X}$ exotic squark.

From the quark self-energy diagrams, one obtains the triangle diagrams needed by attaching a photon or gluon on
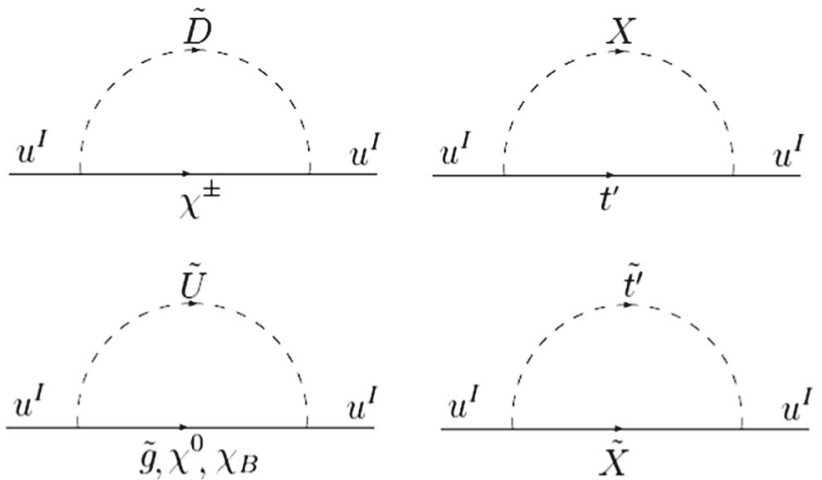

Fig. 1 In the BLMSSM, one-loop self-energy diagrams are collected here, and the corresponding triangle diagrams are obtained from them by attaching a photon or a gluon in all possible ways

the internal lines in all possible ways. After the calculation, we obtain the effective Lagrangian contributing to the quark EDMs and CEDMs. The BLMSSM is larger than MSSM and includes the MSSM contributions. In Fig. 1, we plot all the one-loop self-energy diagrams of the up-type quark.

In this section, we show the one-loop corrections to the quark EDMs (CEDMs). The one-loop chargino-squark contributions are

$$
\begin{aligned}
& d_{\chi_{k}^{ \pm}}^{\gamma}\left(u^{I}\right)=\frac{e \alpha}{4 \pi s_{W}^{2}} V_{U D}^{\dagger} V_{D U} \\
& \times \sum_{i}^{6} \sum_{k}^{2} \operatorname{Im}\left(\left(A_{C}^{D}\right)_{k, i}\left(B_{C}^{D}\right)_{i, k}^{\dagger}\right) \frac{m_{\chi_{k}^{ \pm}}}{m_{\tilde{D}_{i}}^{2}} \\
& \quad \times\left[-\frac{1}{3} \mathcal{B}\left(\frac{m_{\chi_{k}^{ \pm}}^{2}}{m_{\tilde{D}_{i}}^{2}}\right)+\mathcal{A}\left(\frac{m_{\chi_{k}^{ \pm}}^{2}}{m_{\tilde{D}_{i}}^{2}}\right)\right], \\
& d_{\chi_{k}^{ \pm}}^{g}\left(u^{I}\right)=\frac{g_{3} \alpha}{4 \pi s_{W}^{2}} V_{U D}^{\dagger} V_{D U} \\
& \times \sum_{i}^{6} \sum_{k}^{2} \operatorname{Im}\left(\left(A_{C}^{D}\right)_{k, i}\left(B_{C}^{D}\right)_{i, k}^{\dagger}\right) \frac{m_{\chi_{k}^{ \pm}}}{m_{\tilde{D}_{i}}^{2}} \mathcal{B}\left(\frac{m_{\chi_{k}^{ \pm}}^{2}}{m_{\tilde{D}_{i}}^{2}}\right), \\
& \left(A_{C}^{D}\right)_{k, i}=\frac{m_{u^{I}}}{\sqrt{2} m_{W} s_{\beta}}\left(Z_{\tilde{D}}\right)^{J i}\left(Z_{+}\right)^{2 k}, \\
& \left(B_{C}^{D}\right)_{k, i}=\frac{m_{d^{I}}}{\sqrt{2} m_{W} c_{\beta}}\left(Z_{\tilde{D}}\right)^{(J+3) i}\left(Z_{-}\right)^{2 k}-\left(Z_{\tilde{D}}\right)^{J i}\left(Z_{-}\right)^{1 k} .
\end{aligned}
$$

Here $\alpha=\mathrm{e}^{2} /(4 \pi), s_{W}=\sin \theta_{W}, \quad c_{W}=\cos \theta_{W}, \theta_{W}$ is the Weinberg angle, $V$ is the CKM matrix. We define the one-loop functions $\mathcal{A}(r)$ and $\mathcal{B}(r)$ as [27,28]

$$
\begin{aligned}
& \mathcal{A}(r)=\left[2(1-r)^{2}\right]^{-1}[3-r+2 \ln r /(1-r)], \\
& \mathcal{B}(r)=\left[2(r-1)^{2}\right]^{-1}[1+r+2 r \ln r /(1-r)] .
\end{aligned}
$$

$m_{\tilde{D}_{i}}(i=1 \ldots 6)$ are the squark masses and $m_{\chi_{k}^{0}}(k=$ $1,2,3,4)$ denote the eigenvalues of the neutralino mass matrix. $Z_{\tilde{D}_{i}}$ is the rotation matrix used to diagonalize the 
mass squared matrix for the down-type squark. $Z_{-}$and $Z_{+}$ are the rotation matrices used to obtain the mass eigenstates of the charginos.

We show the gluino-squark corrections to the quark EDMs and CEDMs, thus

$$
\begin{aligned}
d_{\tilde{g}}^{\gamma}\left(u^{I}\right)= & -\frac{4}{9 \pi} e \alpha_{s} \sum_{i=1}^{6} \operatorname{Im}\left(\left(\mathcal{Z}_{\tilde{U}}\right)^{(I+3) i}\left(\mathcal{Z}_{\tilde{U}}\right)^{I i *} \mathrm{e}^{-i \theta_{3}}\right) \\
& \times \frac{\left|m_{\tilde{g}}\right|}{m_{\tilde{U}_{i}}^{2}} \mathcal{B}\left(\frac{\left|m_{\tilde{g}}\right|^{2}}{m_{\tilde{U}_{i}}^{2}}\right), \\
d_{\tilde{g}}^{g}\left(u^{I}\right)= & \frac{g_{3} \alpha_{s}}{4 \pi} \sum_{i=1}^{6} \operatorname{Im}\left(\left(\mathcal{Z}_{\tilde{U}}\right)^{(I+3) i}\left(\mathcal{Z}_{\tilde{U}}\right)^{I i *} \mathrm{e}^{-i \theta_{3}}\right) \\
& \times \frac{\left|m_{\tilde{g}}\right|}{m_{\tilde{U}_{i}}^{2}} \mathcal{C}\left(\frac{\left|m_{\tilde{g}}\right|^{2}}{m_{\tilde{U}_{i}}^{2}}\right),
\end{aligned}
$$

with $\alpha_{s}=g_{3}^{2} /(4 \pi) . Z_{\tilde{U}}$ is the matrix for the up-type squarks, with the definition $\mathcal{Z}_{\tilde{U}}^{\dagger} \mathbf{m}_{\tilde{U}}^{2} \mathcal{Z}_{\tilde{U}}=\operatorname{diag}\left(m_{\tilde{q}_{1}}^{2}, \ldots, m_{\tilde{q}_{6}}^{2}\right)$. The concrete form of the loop function $\mathcal{C}(r)$ is $[27,28]$

$\mathcal{C}(r)=\left[6(r-1)^{2}\right]^{-1}[10 r-26-(2 r-18) \ln r /(r-1)]$.

To check the functions $\mathcal{A}(r), \mathcal{B}(r)$ and $\mathcal{C}(r)$ in Refs. [27, 28 ], we calculate the one-loop triangle diagrams using the effective Lagrangian method. In the calculation, we use the approximation

$\frac{1}{(k+p)^{2}-m^{2}}=1-\frac{2 k \cdot p+p^{2}}{k^{2}-m^{2}}+\frac{4(k \cdot p)^{2}}{\left(k^{2}-m^{2}\right)^{2}}$,

with $\mathrm{k}$ representing the loop integral momentum and $\mathrm{p}$ representing the external momentum. It is reasonable because the internal particles are at the order of $\mathrm{TeV}$, and the external quark is lighter than $\mathrm{TeV}$, even for the $t$ quark. The ratio $\frac{m_{t}^{2}}{1 \mathrm{TeV}^{2}} \sim 0.03$ is small enough to use the approximation formula.

For the diagram where the photon is just attached on the internal charged fermions, our result corresponding to $\mathcal{A}(r)$ is the function $a[F, S]$,

$$
\begin{aligned}
a[F, S] & =\frac{\Lambda_{N P}^{2}}{i \pi^{2}} \int \mathrm{d} k^{4} \frac{k^{2}}{\left(k^{2}-m_{F}^{2}\right)^{3}\left(k^{2}-m_{S}^{2}\right)} \\
& =-\frac{F^{2}+2 S^{2} \log (F)-4 F S+3 S^{2}-2 S^{2} \log (S)}{2(F-S)^{3}},
\end{aligned}
$$

with the definition $F=\frac{m_{F}^{2}}{\Lambda_{N P}^{2}}$ and $S=\frac{m_{S}^{2}}{\Lambda_{N P}^{2}} \cdot \lambda_{N P}$ represents the energy scale of the new physics. In order to compare with the function $\mathcal{A}(r)$, we use $\lambda_{N P}^{2}=m_{S}^{2}, S \rightarrow 1, F \rightarrow \frac{m_{F}^{2}}{m_{S}^{2}}=r$ and obtain

$a[r, 1]=-\frac{r^{2}-4 r+2 \log (r)+3}{2(r-1)^{3}}=\mathcal{A}(r)$.

When the photon is just emitted from the internal charged scalars, our result for $\mathcal{B}(r)$ is

$$
\begin{aligned}
b[F, S] & =\frac{\Lambda_{N P}^{2}}{i \pi^{2}} \int \mathrm{d} k^{4} \frac{m_{S}^{2}}{\left(k^{2}-m_{F}^{2}\right)\left(k^{2}-m_{S}^{2}\right)^{3}} \\
& =\frac{F^{2}-2 F S \log (F)+2 F S \log (S)-S^{2}}{2(F-S)^{3}} .
\end{aligned}
$$

With the same approach as that of $a[F, S], b[F, S]$ turns into the form

$$
b[r, 1]=\frac{r^{2}-2 r \log (r)-1}{2(r-1)^{3}}=\mathcal{B}(r) .
$$

$\mathcal{C}(r)$ is obtained from the diagrams where the photon is attached on both the internal charged fermions and the charged scalars. Therefore, $\mathcal{C}(r)$ is the linear combination of $\mathcal{A}(r)$ and $\mathcal{B}(r)$,

$$
\mathcal{C}(r)=\frac{1}{3} \mathcal{B}(r)-3 \mathcal{A}(r) .
$$

From the above discussion, the results in Refs. [27,28] are the same as our results. In our calculation, we do not ignore the mass of the external fermion. So, it is clear that the analytical expressions for the quark EDM in this work are practicable for both $c$ and $t$.

Similarly, the contributions from the one-loop neutralinosquark diagrams are also obtained

$$
\begin{aligned}
d_{\chi_{k}^{0}}^{\gamma}\left(u^{I}\right)= & \frac{e \alpha}{12 \pi s_{W}^{2} c_{W}^{2}} \sum_{i=1}^{6} \sum_{k=1}^{4} \\
& \times \operatorname{Im}\left(\left(A_{N}\right)_{k, i}\left(B_{N}\right)_{i, k}^{\dagger}\right) \frac{m_{\chi_{k}^{0}}}{m_{\tilde{U}_{i}}^{2}} \mathcal{B}\left(\frac{m_{\chi_{k}^{0}}^{2}}{m_{\tilde{U}_{i}}^{2}}\right), \\
d_{\chi_{k}^{0}}^{g}\left(u^{I}\right)= & \frac{g_{3} \alpha}{8 \pi s_{W}^{2} c_{W}^{2}} \sum_{i=1}^{6} \sum_{k=1}^{4} \\
& \times \operatorname{Im}\left(\left(A_{N}\right)_{k, i}\left(B_{N}\right)_{i, k}^{\dagger}\right) \frac{m_{\chi_{k}^{0}}}{m_{\tilde{U}_{i}}^{2}} \mathcal{B}\left(\frac{m_{\chi_{k}^{0}}^{2}}{m_{\tilde{U}_{i}}^{2}}\right), \\
\left(A_{N}\right)_{k, i}= & -\frac{4}{3} s_{W}\left(Z_{\tilde{U}}\right)^{(I+3) i}\left(Z_{N}\right)^{1 k} \\
& +\frac{m_{u^{I}} c_{W}}{m_{W} s_{\beta}}\left(Z_{\tilde{U}}\right)^{I i}\left(Z_{N}\right)^{4 k}, \\
\left(B_{N}\right)_{k, i}= & \left(Z_{\tilde{U}}\right)^{I i}\left(\frac{s_{W}}{3}\left(Z_{N}\right)^{1 k *}+c_{W}\left(Z_{N}\right)^{2 k *}\right) \\
& +\frac{m_{u^{I}} c_{W}}{m_{W} s_{\beta}}\left(Z_{\tilde{U}}\right)^{(I+3) i}\left(Z_{N}\right)^{4 k *} .
\end{aligned}
$$

$Z_{N}$ is the mixing matrix to get the eigenvalues $m_{\chi_{k}^{0}}(k=$ $1,2,3,4)$ of neutralino mass matrix. In the MSSM, there are also the contributions Eqs. (18), (20), and (28). 
At one-loop level, there are three corrections of new type beyond MSSM. The corrections from the virtual $X$ and exotic up-type quark have been deduced in [38-40],

$$
\begin{aligned}
d_{X_{j}}^{\gamma}\left(u^{I}\right)= & \frac{e \lambda_{1} \lambda_{2}}{24 \pi^{2}} \sum_{i, j=1}^{2} \frac{m_{t_{i+3}}}{m_{X_{j}}^{2}} \\
& \times \operatorname{Im}\left(\left(W_{t}\right)_{1 i}\left(Z_{X}\right)_{1 j}^{*}\left(U_{t}\right)_{2 i}^{*}\left(Z_{X}\right)_{2 j}\right) \mathcal{A}\left(\frac{m_{t_{i+3}}^{2}}{m_{X_{j}}^{2}}\right), \\
d_{X_{j}}^{g}\left(u^{I}\right)= & \frac{g_{3} \lambda_{1} \lambda_{2}}{16 \pi^{2}} \sum_{i, j=1}^{2} \frac{m_{t_{i+3}}}{m_{X_{j}}^{2}} \\
& \times \operatorname{Im}\left(\left(W_{t}\right)_{1 i}\left(Z_{X}\right)_{1 j}^{*}\left(U_{t}\right)_{2 i}^{*}\left(Z_{X}\right)_{2 j}\right) \mathcal{A}\left(\frac{m_{t_{i+3}}^{2}}{m_{X_{j}}^{2}}\right),
\end{aligned}
$$

$m_{t_{i+3}}$ and $m_{X_{i}}(i=1,2)$ are mass eigenvalues of the exotic up-type quarks and $X$ superfields. $W_{t}, U_{t}$ and $Z_{X}$ are the mixing matrices defined in Eqs. (6) and (10).

The one-loop baryon neutralino and up-type squark contributions read

$$
\begin{aligned}
d_{\chi_{B}}^{\gamma}\left(u^{I}\right)= & -\frac{e g_{B}^{2}}{108 \pi^{2}} \sum_{i=1}^{2} \sum_{j=1}^{6} \\
& \times \operatorname{Im}\left(\left(Z_{N_{B}}^{1 i}\right)^{2} Z_{\tilde{U}}^{(I+3) j} Z_{\tilde{U}}^{I j *}\right) \frac{m_{\chi_{B}^{i}}}{m_{\tilde{U}_{j}}^{2}} \mathcal{B}\left(\frac{m_{\chi_{B}^{i}}^{2}}{m_{\tilde{U}_{j}}^{2}}\right), \\
d_{\chi_{B}}^{g}\left(u^{I}\right)= & -\frac{g_{3} g_{B}^{2}}{72 \pi^{2}} \sum_{i=1}^{2} \sum_{j=1}^{6} \\
& \times \operatorname{Im}\left(\left(Z_{N_{B}}^{1 i}\right)^{2} Z_{\tilde{U}}^{(I+3) j} Z_{\tilde{U}}^{I j *}\right) \frac{m_{\chi_{B}^{i}}}{m_{\tilde{U}_{j}}^{2}} \mathcal{B}\left(\frac{m_{\chi_{B}^{i}}^{2}}{m_{\tilde{U}_{j}}^{2}}\right) .
\end{aligned}
$$

$m_{\chi_{B}^{i}}(i=1,2,3)$ are the eigenvalues of the baryon neutralino masses.

The exotic up-type squark and $\tilde{X}$ can also contribute to the $c(t) \mathrm{EDM}$ and CEDM

$$
\begin{aligned}
& d_{\tilde{X}}^{\gamma}=\frac{e \lambda_{1} \lambda_{2}}{24 \pi^{2}} \sum_{i=1}^{2} \operatorname{Im}\left(\left(Z_{\tilde{t}^{\prime}}\right)^{3 i *}\left(Z_{\tilde{t}^{\prime}}\right)^{4 i *}\right) \frac{m_{\tilde{X}}}{m_{\tilde{t}_{i+3}}^{2}} \mathcal{B}\left(\frac{m_{\tilde{X}}^{2}}{m_{\tilde{t}_{i+3}}^{2}}\right), \\
& d_{\tilde{X}}^{g}=\frac{g_{3} \lambda_{1} \lambda_{2}}{16 \pi^{2}} \sum_{i=1}^{2} \operatorname{Im}\left(\left(Z_{\tilde{t}^{\prime}}\right)^{3 i *}\left(Z_{\tilde{t}^{\prime}}\right)^{4 i *}\right) \frac{m_{\tilde{X}}}{m_{\tilde{t}_{i+3}}^{2}} \mathcal{B}\left(\frac{m_{\tilde{X}}^{2}}{m_{\tilde{t}_{i+3}}^{2}}\right),
\end{aligned}
$$

with $m_{\tilde{X}}$ and $m_{\tilde{t}_{i+3}}$ denoting the masses of $\tilde{X}$ and exotic uptype squark, respectively.

Using the renormalization group equations $[48,49]$, we evolve the coefficients of the quark EDM and CEDM at matching scale $\mu$ down to the quark $(c, t)$ mass scale,
$d_{q}^{\gamma}\left(\Lambda_{\chi}\right)=1.53 d_{q}^{\gamma}(\Lambda), \quad d_{q}^{g}\left(\Lambda_{\chi}\right)=3.4 d_{q}^{g}(\Lambda)$.

The quark CEDMs can contribute to the quark EDMs at low energy scale. Therefore, they must be taken into account in the numerical calculation and the formula is [50]

$$
d_{c}=d_{c}^{\gamma}+\frac{e}{4 \pi} d_{c}^{g} .
$$

\section{The numerical results}

Here, the results are studied numerically. We take into account not only the experimental constraints from Higgs and neutrino, but also our previous work on this model. From ATLAS collaboration, $m_{\tilde{g}} \geq 1460 \mathrm{GeV}$ is the updated bound on the gluino mass [51]. The parameters are assumed to be

$$
\begin{aligned}
& m_{1}=m_{2}=A_{B Q}=A_{B U}=1 \mathrm{TeV}, \\
& B_{X}=500 \mathrm{GeV}, \quad m_{D}^{2}=\delta_{i j} \mathrm{TeV}^{2}, \quad(i, j=1,2,3), \\
& A_{u}=A_{d}=A_{u}^{\prime}=A_{d}^{\prime}=500 \mathrm{GeV}, \\
& Y_{d_{4}}=Y_{d_{5}}=0.7 Y_{b}, \quad \lambda_{Q}=\lambda_{u}=0.5, \\
& m_{\tilde{Q}_{4}}^{2}=m_{\tilde{Q}_{5}}^{2}=m_{\tilde{U}_{4}}^{2}=m_{\tilde{U}_{5}}^{2}=1 \mathrm{TeV}^{2}, \\
& B_{4}=\frac{3}{2}, \quad A_{u_{4}}=A_{u_{5}}=500 \mathrm{GeV} .
\end{aligned}
$$

\section{$4.1 c$ quark EDM}

For the $c$ quark EDM, we use the following parameters:

$$
\begin{aligned}
& \tan \beta=10, \quad \mu=800 \mathrm{GeV}, \quad m_{\tilde{g}}=1600 \mathrm{GeV}, \\
& \tan \beta_{B}=2, \quad v_{B_{t}}=3 \mathrm{TeV}, \quad Y_{u_{4}}=Y_{u_{5}}=0.7 Y_{t} .
\end{aligned}
$$

The baryon neutralino and squarks can give contributions to the $c$ quark EDM, which is relevant to the parameters $g_{B}$ and $m_{B} \cdot m_{B}$, representing baryon gaugino masses, can have a nonzero CP-violating phase $\theta_{m_{B}}$. Both $m_{B}$ and $g_{B}$ influence the baryon neutralino masses. Furthermore, $g_{B}$ is the coupling constant for the quark-squark-baryon neutralino. So, with $\theta_{m_{B}}=-0.5 \pi, \lambda_{1}=\lambda_{2}=0.1, \mu_{X}=\mu_{B}=3 \mathrm{TeV}$, $m_{Q}^{2}=m_{U}^{2}=\delta_{i j} \mathrm{TeV}^{2}$ for $(i, j=1,2,3)$, we study the $c$ quark EDM versus $g_{B}$. If we do not mention the other CPviolating phases, it indicates the other $\mathrm{CP}$-violating phases are zero. In Fig. 2, the numerical results corresponding to $m_{B}=(1,2,3) \times \mathrm{e}^{i \theta_{m_{B}}} \mathrm{TeV}$ are plotted by the solid line, dotted line, and dashed line, respectively. On the whole, the three lines are all increasing functions of $g_{B}$. The dotted line and the dashed line vary slightly. The solid line quickly gets larger with increasing $g_{B}$. These three lines also imply the results are suppressed by large $\left|m_{B}\right|$. The obtained numerical results from the nonzero CP-violating phase $\theta_{m_{B}}$ are at the order of $10^{-21} \mathrm{e} \mathrm{cm}$, four orders smaller than the upper bound of the $c$ quark EDM. 


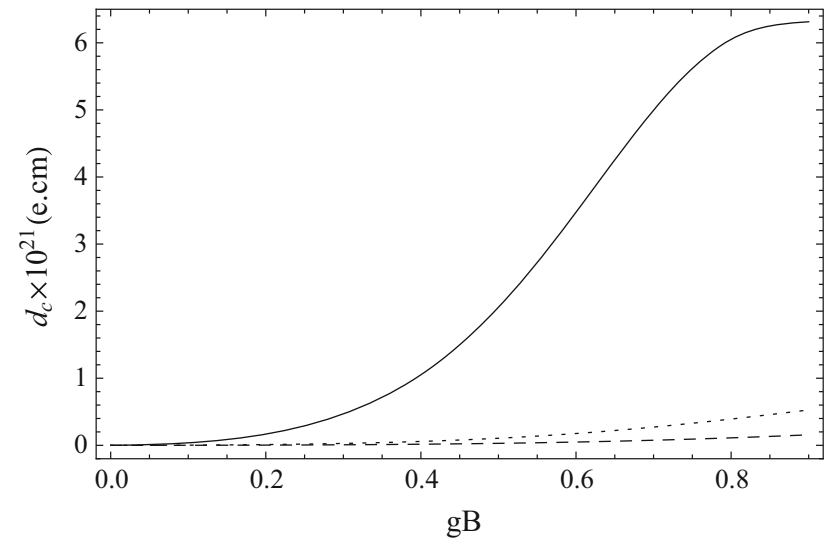

Fig. 2 The one-loop corrections to the $c$ EDM versus gB with $\theta_{m_{B}}=$ $-0.5 \pi$, the solid line, dotted line, and dashed line corresponding to $m_{B}=(1,2,3) \times \mathrm{e}^{\mathrm{i} \theta_{m_{B}}} \mathrm{TeV}$, respectively

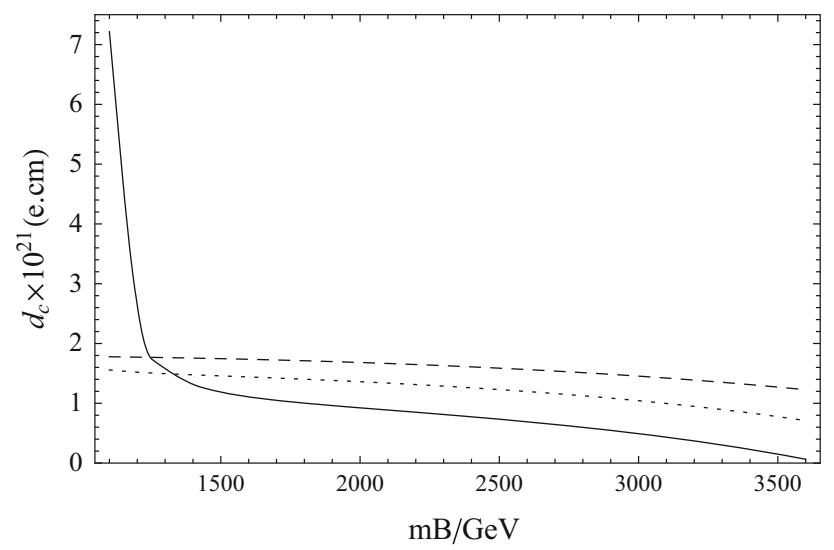

Fig. 3 The one-loop corrections to the $c$ EDM versus $\mathrm{mB}$ with $\theta_{\mu_{B}}=$ $0.5 \pi$, the solid line, dotted line and dashed line corresponding to $g_{B}=$ $(1 / 3,1 / 5,1 / 10)$, respectively

Here, we discuss the effects from the new phase $\theta_{\mu_{B}}$ of $\mu_{B}$; it may influence the baryon neutralino masses. Based on the supposition $\mu_{B}=2 \times \mathrm{e}^{\mathrm{i} \theta_{\mu_{B}}} \mathrm{TeV}\left(\theta_{\mu_{B}}=0.5 \pi\right), \quad \lambda_{1}=$ $\lambda_{2}=0.5, \quad \mu_{X}=3 \mathrm{TeV}, m_{Q}^{2}=4 \delta_{i j} \mathrm{TeV}^{2}, m_{U}^{2}=$ $2 \delta_{i j} \mathrm{TeV}^{2}(i, j=1,2,3)$, the results versus $m_{B}$ are shown as the solid line for $g_{B}=\frac{1}{3}$. The solid line decreases quickly in the region $1000 \mathrm{GeV}<m_{B}<1300 \mathrm{GeV}$. When $m_{B}>1300 \mathrm{GeV}$, the extent of the change of the solid line is small. The dotted line and the dashed line, respectively, represent the results for $g_{B}=\frac{1}{5}$ and $g_{B}=\frac{1}{10}$, and they are both slowly decreasing functions of $m_{B}$. Generally speaking, the results are around $10^{-21} \mathrm{e} \mathrm{cm}$, at the same order as of Fig. 2.

$\lambda_{1}$ and $\lambda_{2}$ are important parameters for the couplings of quark-exotic quark- $X$ and quark-exotic squark- $\tilde{X}$. Therefore, the numerical results may obviously be influenced by the varying $\lambda_{1}$ and $\lambda_{2}$. For simplicity, we suppose $\lambda_{1}=\lambda_{2}=$ Lam, $g_{B}=\frac{1}{3}, m_{B}=1 \mathrm{TeV}, \mu_{B}=3 \mathrm{TeV}, m_{Q}^{2}=m_{U}^{2}=$ $\delta_{i j} \mathrm{TeV}^{2}$ for $(i, j=1,2,3)$. With the nonzero CP-violating phase $\theta_{X}=(0.5 \pi, 0.3 \pi, 0.1 \pi)$ and $\mu_{X}=\mathrm{e}^{i \theta_{X}} \mathrm{TeV}$, the

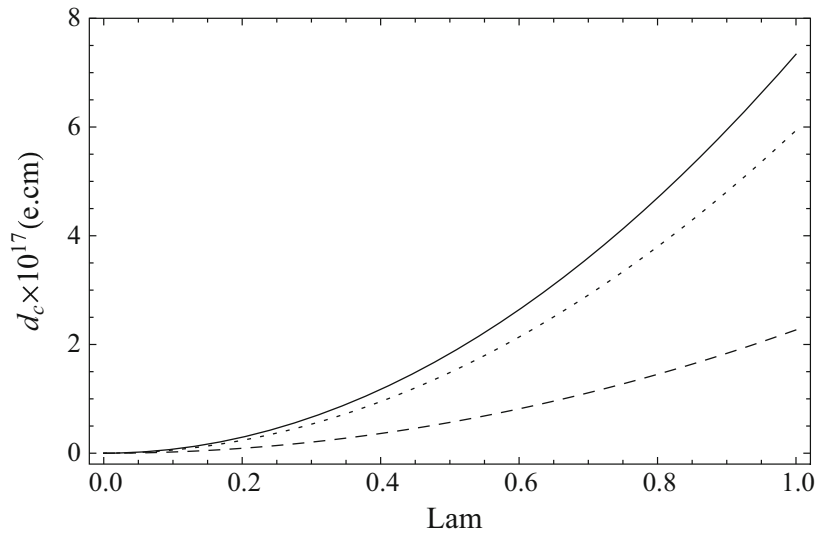

Fig. 4 The one-loop corrections to the $c$ EDM versus Lam, while the solid line, dotted line and dashed line denoting the results with $\mu_{X}=$ $\mathrm{e}^{i(0.5 \pi, 0.3 \pi, 0.1 \pi)} \mathrm{TeV}$, respectively

results versus Lam are denoted, respectively, by the solid line, dotted line, and dashed line. The three lines are the increasing functions of Lam and the results are around $10^{-17} \mathrm{e} \mathrm{cm}$. As Lam $>0.8$, the solid line even exceeds the upper bound $5.0 \times 10^{-17}$ e cm. From Figs. 2, 3, and 4, one can see that the effects from $\theta_{X}$ are much larger than the effects from $\theta_{\mu_{B}}$ and $\theta_{m_{B}}$.

\section{$4.2 t$ quark EDM}

To calculate the $t$ quark EDM numerically, we use the parameters

$$
g_{B}=\frac{1}{3}, \quad m_{B}=1 \mathrm{TeV}, \quad \mu_{B}=3 \mathrm{TeV} .
$$

The quark-gluino-squark coupling corrections to the $t$ quark EDM are shown in Eq. (20). $\tan \beta$ is important, because it influences the masses of chargino, neutralino, squark, and so on. The mixing matrices of squarks and exotic squarks have a relation with $\tan \beta$. The absolute value of the gluino mass obviously also influences the results from Eq. (20). Using the parameters $\theta_{3}=-0.5 \pi, \mu=800 \mathrm{GeV}, \tan \beta_{B}=$ 2, $Y_{u_{4}}=Y_{u_{5}}=0.7 Y_{t}, \lambda_{1}=\lambda_{2}=0.5, \mu_{X}=1 \mathrm{TeV}, V_{B_{t}}=$ $3300 \mathrm{GeV}, m_{Q}^{2}=\delta_{i j} 1500^{2} \mathrm{GeV}^{2}, m_{U}^{2}=\delta_{i j} \mathrm{TeV}^{2}$ with $(i, j=1,2,3)$, for the $t$ quark EDM we plot the results versus $m_{\tilde{g}}$. In Fig. 5 , the solid line $(\tan \beta=5)$, dotted line $(\tan \beta=10)$ and dashed line $(\tan \beta=15)$ are all decreasing functions of $m_{\tilde{g}}$. During the $m_{\tilde{g}}$ region (1500-2000) GeV, the results of the three lines shrink quickly. Near the point $m_{\tilde{g}}=1480 \mathrm{GeV}$, the theoretical predictions are of the order of $10^{-16} \mathrm{e} \mathrm{cm}$ and even reach $10^{-15} \mathrm{e} \mathrm{cm}$. On the other hand, the extent of the influence from $\tan \beta$ is not large.

The effects to the $t$ quark EDM from the $\mu$ parameter are also of interest. $\mu$ is included in the mass matrices of chargino and neutralino. On the other hand $m_{Q}^{2}$ and $m_{u}^{2}$ can affect the masses and mixings of the up-type squark. For simplification 


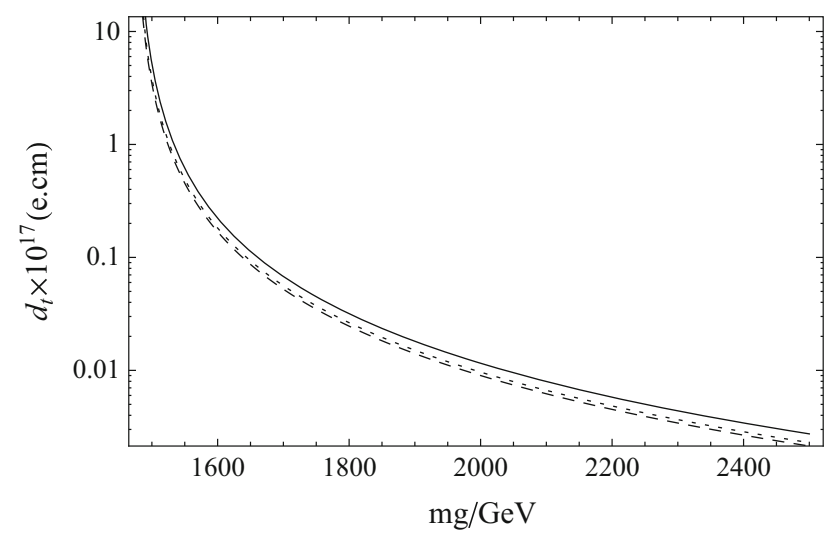

Fig. 5 The one-loop corrections to the $t$ EDM versus $m_{\tilde{g}}$ with $\theta_{3}=$ $-0.5 \pi$, the solid line, dotted line, and dashed line corresponding to $\tan \beta=(5,10,15)$

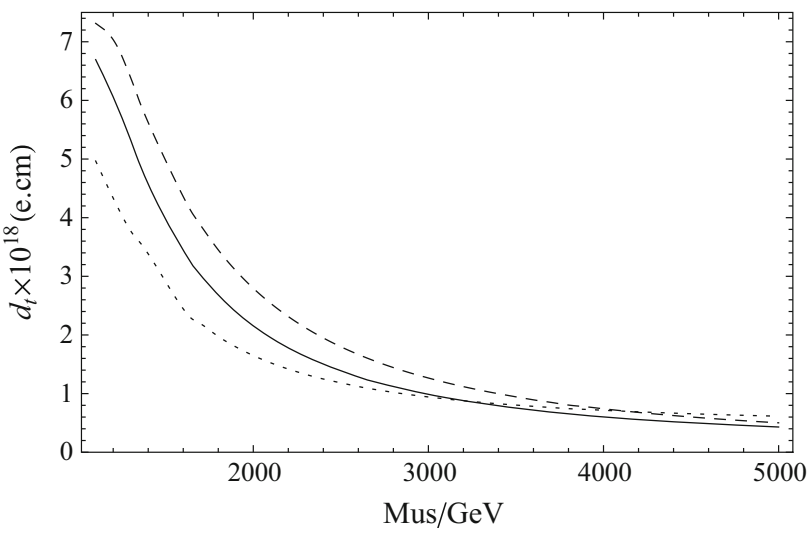

Fig. 6 The one-loop corrections to the $t$ EDM versus Mus with $\theta_{\mu}=$ $-0.5 \pi$, the dotted line, solid line, and dashed line corresponding to the results with $\mu=(1500,2500,3500) \mathrm{e}^{i \theta_{\mu}} \mathrm{GeV}$

of the numerical discussion, we adopt the relation $m_{Q}^{2}=$ $m_{U}^{2}=M u s^{2}$ and use the parameters $\tan \beta=10, m_{\tilde{g}}=$ $1600 \mathrm{GeV}, \tan \beta_{B}=1.5, V_{B_{t}}=3600 \mathrm{GeV}, Y_{u_{4}}=Y_{u_{5}}=$ $0.7 Y_{t}, \lambda_{1}=\lambda_{2}=0.1, \mu_{X}=1 \mathrm{TeV}$. As $\theta_{\mu}=-0.5 \pi$ and $\mu=(1500,2500,3500) \mathrm{e}^{i \theta_{\mu}} \mathrm{GeV}$, the numerical results for the $t$ quark EDM are plotted, respectively, by the dotted line, solid line, and dashed line. The results are all decreasing functions of Mus and at the order of $10^{-18} \mathrm{e} \mathrm{cm}$ as Mus $<3500 \mathrm{GeV}$. The absolute value of $\mu$ also influences the results, and the extent is small (Fig. 6).

The exotic squarks and exotic quarks are in connection with $Y_{u_{4}}$ and $Y_{u_{5}}$, and the related contributions are shown in Eqs. $(29,31)$. As discussed in a previous subsection, nonzero $\theta_{X}$ can give large contributions. In Fig. 7 we plot the solid line, dotted line, and dashed line versus $Y u 45$ with $Y_{u_{4}}=Y_{u_{5}}=Y u 45 * Y t, \quad \theta_{X}=(0.5 \pi, 0.2 \pi, 0.05 \pi)$ and $\mu_{X}=1 \mathrm{e}^{i \theta_{X}} \mathrm{TeV}$. The other parameters used are $\tan \beta=10, \mu=800 \mathrm{GeV}, m_{\tilde{g}}=1600 \mathrm{GeV}, \tan \beta_{B}=$ 2, $V_{B_{t}}=3 \mathrm{TeV}, \lambda_{1}=\lambda_{2}=0.5, m_{Q}^{2}=m_{U}^{2}=\delta_{i j} \mathrm{TeV}^{2}$ with $(i, j=1,2,3)$. They are all increasing functions of

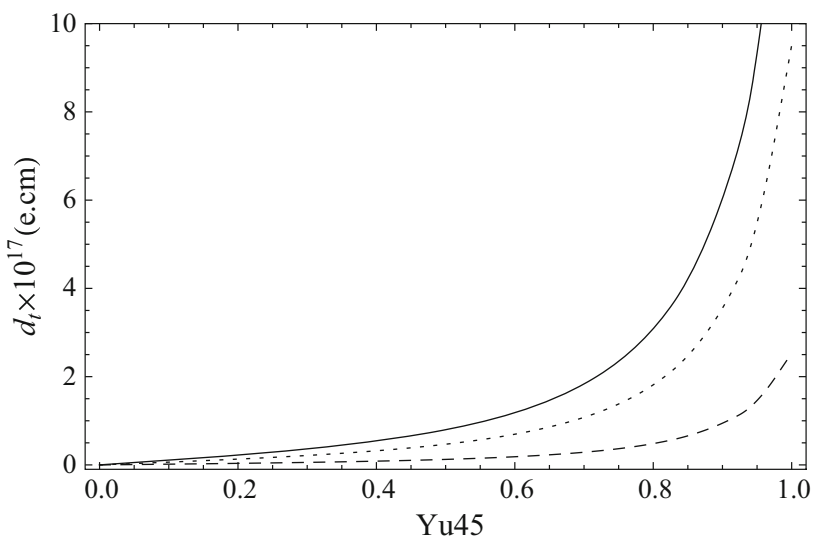

Fig. 7 The one-loop corrections to the $t$ EDM versus Yu45 with $\mu_{X}=$ $\mathrm{e}^{i \theta_{X}} \mathrm{TeV}$, the solid line, dotted line, and dashed line corresponding to the results with $\theta_{X}=(0.5 \pi, 0.2 \pi, 0.05 \pi)$

$Y u 45$ and as $Y u 45>0.8$ the numerical results get quickly larger. At the point $Y u 45=0.7$, the solid line and dotted line are larger than $1 \times 10^{-17} \mathrm{e} \mathrm{cm}$. The three lines are at the order of $10^{-18} \mathrm{e} \mathrm{cm}$, when $Y u 45$ is small. For the $c$ and $t$ quark EDMs, in our parameter space the CP-violating phases $\theta_{3}$ and $\theta_{X}$ are important and can provide large contributions.

\section{Discussion}

In the CP-violating BLMSSM, there are new CP-violating phases $\theta_{X}, \theta_{\mu_{B}}, \theta_{m_{B}}$ beyond MSSM. For the c quark EDM, we consider the conditions $\theta_{X} \neq 0, \theta_{\mu_{B}} \neq 0$ and $\theta_{m_{B}} \neq 0$, respectively. The results show $\theta_{X}$ can give large contributions, even reach the experimental upper bound $\left(5 \times 10^{-17} \mathrm{e}\right.$ $\mathrm{cm}$ ) for the $c$ EDM. The effects produced from $\theta_{m_{B}}$ and $\theta_{\mu_{B}}$ are at the order of $10^{-21} \mathrm{e} \mathrm{cm}$, which are much smaller than those from $\theta_{X}$. For the $t$ quark EDM the CP-violating phases $\theta_{3}, \theta_{\mu}$, and $\theta_{X}$ are studied. Under the two conditions $\theta_{X} \neq 0$ and $\theta_{3} \neq 0$, we find $d_{t}$ to be at the order of $10^{-17} \mathrm{e} \mathrm{cm}$. Especially for nonzero $\theta_{3}$ with $m_{\tilde{g}}$ near its lower bound, the $t$ quark EDM can reach $10^{-16} \mathrm{e} \mathrm{cm}$ and even higher. They are both larger than the results for $\theta_{\mu} \neq 0$.

In BLMSSM, at one-loop level there are contributions of three types [(1) the virtual $X$ and exotic up-type quark, (2) baryon neutralino and up-type squark, (3) exotic up-type squark and $\tilde{X}]$ to the quark EDM beyond MSSM. For the contributions beyond MSSM, to obtain large $d_{c}$ and $d_{t}$ the CP-violating phase $\theta_{X}$ should be nonzero in our parameter space. With only $\theta_{X} \neq 0$, the nonzero contributions come from Eqs. (23) and (25). In Fig. $4 d_{c} \sim 10^{-17} \mathrm{e} \mathrm{cm}$ and in Fig. $7 d_{t} \sim 10^{-17} \mathrm{e} \mathrm{cm}$, the EDMs $d_{c}$ and $d_{t}$ are of the same order of magnitude. In the other work, the EDMs $d_{c}$ and $d_{t}$ should be of different order. What is the reason in this work? The reason can be found from the couplings in Eqs. (13) 
and (14). The coupling constants $\lambda_{1}$ and $\lambda_{2}$ are important parameters. In our numerical calculation, we adopt the case that the values of $\lambda_{1}\left(\lambda_{2}\right)$ for $c$ quark are the same as the values of $\lambda_{1}\left(\lambda_{2}\right)$ for $t$ quark. That is to say, for the up-type quark generation 2 and generation 3 , the adopted values of $\lambda_{1}\left(\lambda_{2}\right)$ are the same.

From these numerical results and our previous work on the neutron EDM, we find that $\theta_{3}$ and $\theta_{X}$ are important CPviolating phases. $\tan \beta, Y_{u_{4}}, Y_{u_{5}}, m_{\tilde{g}}, m_{Q}^{2}, m_{U}^{2}, \lambda_{1}, \lambda_{2}, V_{B_{t}}$ are also important. On the whole, our numerical results are sufficiently large so as to be detected in the future. The work can confine the parameter space in this model and has significance for the relevant experiments for the $c$ and $t$ quark EDMs.

Acknowledgements The work has been supported by the National Natural Science Foundation of China (NNSFC) with Grant Nos. 11275036, 11535002, 11605037, 11647120, the Fund of Natural Science Foundation of Hebei Province (A2011201118), Natural Science Fund of Hebei University with Grants Nos. 2011JQ05, 2012-242. Hebei Key Lab of Optic-Electronic Information and Materials, the midwest universities comprehensive strength promotion project.

Open Access This article is distributed under the terms of the Creative Commons Attribution 4.0 International License (http://creativecomm ons.org/licenses/by/4.0/), which permits unrestricted use, distribution, and reproduction in any medium, provided you give appropriate credit to the original author(s) and the source, provide a link to the Creative Commons license, and indicate if changes were made. Funded by SCOAP ${ }^{3}$.

\section{References}

1. J.M. Christensen, J.W. Cronin, V.L. Fitch, R. Turlay, Phys. Rev. Lett. 13, 138 (1964)

2. K. Abe et al., Belle Collaboration, Phys. Rev. Lett. 87, 091802 (2001)

3. B. Aubert et al., BaBar Collaboration, Phys. Rev. Lett. 89, 201802 (2002)

4. J. Ellis, S. Ferrara, D.V. Nanopoulos, Phys. Lett. B 114, 231 (1982)

5. J. Polchinski, M.B. Wise, Phys. Lett. B 125, 393 (1983)

6. P. Nath, Phys. Rev. Lett. 66, 2565 (1991)

7. Y. Kizukuri, N. Oshimo, Phys. Rev. D 46, 3025 (1992)

8. Y. Kizukuri, N. Oshimo, Phys. Rev. D 45, 1806 (1992)

9. E. Shabalin, Sov. J. Nucl. Phys. 28, 75 (1978)

10. F. Sala, JHEP 1403, 061 (2014)

11. I. Khriplovich, Phys. Lett. B 173, 193-196 (1986)

12. A. Czarnecki, B. Krause, Phys. Rev. Lett. 78, 4339-4342 (1997)

13. CMS Collaboration, Phys. Lett. B 716, 30 (2012)

14. ATLAS Collaboration, Phys. Lett. B 716, 1 (2012)
15. J. Rosiek, Phys. Rev. D 41, 3464 (1990). arXiv:hep-ph/9511250

16. H.P. Nilles, Phys. Rep. 110, 1 (1984)

17. H.E. Haber, G.L. Kane, Phys. Rep. 117, 75-263 (1985)

18. N. Escudero, D.E. Lopez-Fogliani, C. Munoz, R.R. de Austri, JHEP 12, 099 (2008)

19. Hai-Bin Zhang, Tai-Fu Feng, Fei Sun et al., Phys. Rev. D 89, 115007 (2014)

20. A. Pilaftsis, Phys. Rev. D 58, 096010 (1998)

21. M. Carena, J. Ellis, A. Pilaftsis, C.E.M. Wagner, Nucl. Phys. B 586, $92(2000)$

22. Tai-Fu Feng, Lin Sun, Xiu-Yi Yang, Nucl. Phys. B 800, 221 (2008)

23. Tai-Fu Feng, Lin Sun, Xiu-Yi Yang, Phys. Rev. D 77, 116008 (2008)

24. I. Altarev et al., Phys. Lett. B 276, 242 (1992)

25. K. Smith et al., Phys. Lett. B 234, 191 (1990)

26. C. Patrignani et al., Particle Data Group, Chin. Phys. C 40, 100001 (2016)

27. T. Ibrahim, P. Nath, Phys. Rev. D 57, 478 (1998) [Erratum. Phys. Rev. D 58, 019901 (1998)]

28. T. Ibrahim, P. Nath, Phys. Lett. B 418, 98 (1998) [Erratum: Phys. Lett. B 460, 498 (1999)]

29. P.F. Perez, M.B. Wise, JHEP 1108, 068 (2011)

30. P.F. Perez, M.B. Wise, Phys. Rev. D 82, 011901 (2010)

31. P.F. Perez, Phys. Lett. B 711, 353 (2012)

32. J.M. Arnold, P.F. Perez, B. Fornal, S. Spinner, Phys. Rev. D 85, 115024 (2012)

33. P.F. Perez, M.B. Wise, Phys. Rev. D 84, 055015 (2011)

34. Tai-Fu Feng, Shu-Min Zhao, Hai-Bin Zhang, Yin-Jie Zhang, Yu-Li Yan, Nucl. Phys. B 871, 223 (2013)

35. S.M. Zhao, T.F. Feng, H.B. Zhang et al., JHEP 11, 119 (2014)

36. S.M. Zhao, T.F. Feng, H.B. Zhang et al., Phys. Rev. D 92, 115016 (2015)

37. S.-M. Zhao, T.-F. Feng, X.-X. Dong et al., Nucl. Phys. B 910, 225-239 (2016)

38. S.M. Zhao, T.F. Feng, B. Yan et al., JHEP 10, 020 (2013)

39. F. Sun, T.F. Feng, S.M. Zhao et al., Nucl. Phys. B 888, 30 (2014)

40. S.M. Zhao, T.F. Feng, X.J. Zhan et al., JHEP 07, 124 (2015)

41. A. Cordero-Cid, J.M. Hernandez, G. Tavares-Velasco, J.J. Toscano, J. Phys. G 35, 025004 (2008)

42. A.G. Grozin, I.B. Khriplovich, A.S. Rudenko, Nucl. Phys. B 821, 285-290 (2009)

43. A.E. Blinov, A.S. Rudenko, Nucl. Phys. Proc. Suppl. 189, 257-259 (2009)

44. Filippo Sala, JHEP 1403, 061 (2014)

45. Z.Z. Aydin, U. Erkarslan, Phys. Rev. D 67, 036006 (2003)

46. Xing-Xing Dong, Shu-Min Zhao, Hai-Bin Zhang et al., Chin. Phys. C 40, 093103 (2016)

47. Tai-Fu Feng, Xue-Qian Li, Jukka Maalampi, Xin-min Zhang, Phys. Rev. D 71, 056005 (2005)

48. R. Arnowitt, J. Lopez, D.V. Nanopoulos, Phys. Rev. D 42, 2423 (1990)

49. R. Arnowitt, M. Duff, K. Stelle, Phys. Rev. D 43, 3085 (1991)

50. A. Manohar, H. Georgi, Nucl. Phys. B 234, 189 (1984)

51. M. Aaboud et al., ATLAS Collaboration, Phys. Rev. D 94, 052009 (2016) 